\title{
Nonlinear ICRF-Plasma Interactions
}

\author{
J. R. Myra, D. A. D'Ippolito and D.A. Russell \\ Lodestar Research Corp., Boulder, Colorado 80301 \\ L. A. Berry, E. F. Jaeger and M. D. Carter \\ Oak Ridge National Laboratory, Oak Ridge, Tennessee 37831
}

\begin{abstract}
April 2005
submitted to Proceedings of the 16th Topical Meeting on RF Power in Plasmas, Park City, Utah USA, April 11-13, 2005
\end{abstract}

\section{LODESTAR RESEARCH CORPORATION 2400 Central Avenue Boulder, Colorado 80301}




\title{
Nonlinear ICRF-Plasma Interactions
}

\author{
J. R. Myra, D. A. D’Ippolito, and D.A. Russell \\ Lodestar Research Corporation \\ Boulder, Colorado, USA \\ L. A. Berry, E. F. Jaeger, and M. D. Carter \\ Oak Ridge National Laboratory \\ Oak Ridge, Tennessee, USA
}

\begin{abstract}
The well developed linear theory of ICRF (including FW, HHFW and IBW) interactions with plasma has enjoyed considerable success in describing antenna coupling and wave propagation, and provides a well-known framework for calculating power absorption, current drive, etc. In some situations, less well studied nonlinear effects are of interest, such as flow drive, ponderomotive forces, rf sheaths, parametric decay and related interactions with the edge plasma. Standard ICRF codes have begun to integrate this physics to achieve improved modeling capabilities. This paper concentrates on basic rf-plasma-interaction physics with illustrative applications to tokamaks. For FW antennas, the parallel electric field near launching structures is known to drive rf-sheaths which can give rise to convective cells, interaction with plasma "blobs", impurity production, and edge power dissipation. In addition to sheaths, IBW waves in the edge plasma are subject to strong ponderomotive effects and parametric decay. In the core plasma, slow waves can sometimes induce nonlinear effects. Mechanisms by which these waves can influence the radial electric field and its shear are summarized, and related to the general (reactive-ponderomotive and dissipative) force on a plasma from rf waves. It is argued that there are significant opportunities now for new predictive capabilities by advances in integrated simulation.
\end{abstract}

Keywords: nonlinear, ion cyclotron range, sheath, ponderomotive, parametric, flow, shear. PACS: $52.40 . \mathrm{Kh}, 52.35 . \mathrm{Mw}, 52.40 . \mathrm{Fd}$

\section{INTRODUCTION}

RF physics is arguably one of the richest areas of plasma physics in which linear theory is both widely applicable to experiment and exceedingly rich in the complexity and subtlety of physical phenomena which it can describe. This fact underlies many decades of successful theoretical research in ion cyclotron range of frequencies (ICRF) wave physics. Nevertheless, there are a few instances when linear physics fails, and nonlinear effects can become important. The most obvious case is near the antenna where the rf fields are large, and typical rf voltages, and/or ponderomotive potentials easily exceed the local plasma temperature $\mathrm{T}_{\mathrm{e}}$. Wave-induced rf sheath voltages can also exceed $\mathrm{T}_{\mathrm{e}}$ near walls and limiters where the plasma temperature is low. In addition to these situations in the edge plasma, nonlinear effects can also be important 
further in towards the core for slow waves, which have a small group velocity and therefore require a large electric field to carry power. In these cases, nonlinear plasma interactions can drive plasma flows and radial electric field shear, of interest for turbulence suppression and transport barrier formation.

The goal of this paper is to summarize physics concepts in these areas where nonlinear effects enter, indicate available modeling and analysis tools, and point out opportunities for new predictive capabilities. Although experimental motivation will be given, this paper will not attempt a comprehensive experimental review. Many of the relevant older experimental references have been given elsewhere. ${ }^{1-3}$

The outline of our paper is as follows. In the next section we consider antennaedge interactions arising in fast wave (FW) launch from rf sheaths. This is followed by a discussion of additional effects present in ion Bernstein wave (IBW) direct launch experiments. Then IBW and ion cyclotron wave (ICW) core interactions and driven flows are reviewed. The final section presents some thoughts on the opportunities and prospects for future theoretical work involving integrated computer modeling.

\section{FW LAUNCH ANTENNA-EDGE INTERACTIONS: RF SHEATHS}

A variety of rf edge interactions have been seen on ICRF experiments for several decades.1,2 These rf specific effects include: impurities (RF-enhanced sputtering), density rise, arcs, antenna damage, and anomalous edge power dissipation. RF antenna voltage controls the near field rf-specific effects, which are generally least severe in anti-symmetric (e.g. dipole) phasing. ${ }^{4}$ Experience and intuition developed over the years have partly, if not mostly mitigated deleterious effects in many experiments, under most circumstances. But, it is likely that they will have important implications for present and future long pulse operation, where even small effects can have large consequences. 5

The primary culprit for many of the observed phenomena is the rf sheath which exists at "end-plates" where the field line contacts a conductor. Important places where rf sheaths occur will be identified later. The basic physics underlying a sheath is that both species, electrons and ions, initially try to leave at their respective thermal velocities. In response to the growing charge imbalance, the plasma develops a potential to confine the electrons. This potential, which must be higher than the applied voltage at either of the two ends, reflects almost all of the electrons at the sheath entrance. The sheath width $\Delta \sim \lambda_{\mathrm{d}}\left(\mathrm{eV} / \mathrm{T}_{\mathrm{e}}\right)^{3 / 4}$ at each end is determined by requiring that the un-neutralized ion space charge in the sheath layer is sufficient to give rise to the requited potential drop. Here $\lambda_{\mathrm{d}}$ is the Debye length, e the proton charge, $\mathrm{V}$ the applied voltage and $\mathrm{T}_{\mathrm{e}}$ the electron temperature. In addition to reflecting electrons, this large sheath accelerates ions into the plates causing sputtering. The energy for this accelerations comes from the circuit, and appears as lost power to the sheath. Thus, the whole process is driven by the need for charge ambipolarity.

This basic sheath physics extends immediately into an ICRF sheath, ${ }^{6-9}$ where an oscillating voltage is applied to each plate. Electrons leave alternately out one end, then the other, escaping from the end where applied voltage is highest (and hence the reflecting barrier seen by the electrons lowest). This give rise to an oscillating parallel 
electron current. The central voltage oscillates up and down at twice the applied frequency, but always remains higher that the applied voltage at either end. The net effect is that there is both rectification of the applied voltage and a large second harmonic. The net sheath power dissipation (for the case of Maxwell-Boltzmann electrons) is given by 10,11

$$
\mathrm{P}_{\mathrm{sh}}=\mathrm{n}_{\mathrm{e}} \mathrm{C}_{\mathrm{s}} \mathrm{AT}_{\mathrm{e}} \xi \mathrm{I}_{1}(\xi) / \mathrm{I}_{0}(\xi) \rightarrow \mathrm{n}_{\mathrm{e}} \mathrm{C}_{\mathrm{S}} \mathrm{AeV}_{\mathrm{rf}}
$$

where $n_{e} c_{s}$ is the plasma flux, $A$ is the projected area normal to the magnetic field, $\xi=$ $\mathrm{eV}_{\mathrm{rf}} / \mathrm{T}_{\mathrm{e}}, \mathrm{I}_{0}$ and $\mathrm{I}_{1}$ are Bessel functions, and the final form is the high voltage limit. The two most important parameters are the plasma density (and flux) into the antenna, and the rf voltage.

Several other considerations are important, including the angle at which the field line strikes the plate. This influences the ion orbits in the sheath, and impacts surface physics calculations such as sputtering. Simulations have been performed to quantify these and other effects.7,8 In particular, for shallow incidence of the field lines onto the surface, there is a significant magnetic presheath in which the ion flow transitions from being sonic along the field lines to sonic normal to the plate. ${ }^{8}$ These types of calculations confirm that the sheath voltage drop available for ion acceleration and power dissipation is normally an order unity fraction of the applied rf voltage, because the potential drop in the sheaths is largely controlled by electron physics and simple ambipolarity considerations.

One of the goals that is being pursued in contemporary research is that of including plasma and rf-sheath effects in antenna coupling codes. Since it is still impractical to do full wave particle simulations for the rf fields and sheaths, it is important to be able to characterize the main effect of the sheaths in a simple way. A useful model ${ }^{12}$ is to regard the electrons as an oscillating charge layer, which leaves a vacuum gap in the rf sheath. As far as the rf is concerned, this vacuum gap provides an extra capacitance in the $\mathrm{rf}$ circuit. This type of model was investigated ${ }^{13}$ for plasma processing and is currently being tested in an rf antenna coupling code for fusion applications. ${ }^{14}$ The presence of rf sheaths can modify the rf-field distribution between antenna bumper limiters.

Utilizing the fact that the sheath is a thin layer, it is also possible to analytically derive a "sheath boundary condition" that can replace the usual perfect conductor boundary conditions on the tangential component of $\mathrm{E}$ and the normal component of $\mathrm{B}$ on the surface of a conductor. ${ }^{15,16}$ The resulting boundary condition involves the sheath width and its effective dielectric properties which can model both sheath capacitance and dissipation (resistance).

The interesting and important question concerning self-consistent rf coupling codes and sheath interactions is the competition associated with the plasma density near the antenna. A large density is favorable for good coupling (because the launched FW is usually evanescence at low densities) but this also increases the level of sheath interaction. These considerations are partly addressable in antenna design, for example by using septa and bumper limiters. ${ }^{17}$

RF sheaths occur where field lines containing plasma contact conducting surfaces. On the antenna itself, the geometry of these connections implies a phasing and field line angle dependence. ${ }^{18}$ For a two-strap antenna in $0-\pi$ (dipole) phasing, and 
symmetrical sheath connections on the front face of the Faraday screen, there is no net rf voltage induced between contact points. However in 0-0 (monopole) phasing a large voltage can result. The voltage in the case is essentially the fraction of the end-to-end voltage along the current strap that is subtended by the contacts.

This kind of Faraday screen rf sheath is exacerbated by large misalignment of the B-field with the Faraday Screen and/or a large component of B along the current strap, and by low- $\mathrm{k}_{\|}$non-symmetric phasings. As a result, experiments have shown that ICRF heating in dipole phasing is much easier than ICRF current drive.

In practice, not all important sheaths are this simple: capacitive, corner and feeder effects also drive rf sheaths. ${ }^{19}$ While the sheath voltages in 0 - $\pi$ phasing are smaller, they are not zero. One reason is that the field lines that cut across the corner of the antenna don't see anti-symmetric, canceling rf magnetic fluxes. A related effect is that current forced onto the current straps by the feeders leads to charges where the conductors take a sharp corner, and this can be thought of as a capacitive coupling to the rf sheaths.

Due to the grazing nature of the field lines contacting the complicated threedimensional structure of an antenna, the contact points are very sensitive to field line location, and adjacent field lines can end up having very different induced sheath voltages because their induction circuits trap a different amount of rf magnetic flux. When adjacent field lines charge to different voltages, there is a perpendicular electric field between them. This gives rise to $\mathbf{E} \times \mathbf{B}$ drifts and the important concept of rfsheath-induced convection. ${ }^{20-22}$

The effects of rf-induced convection have been seen indirectly in experiments. On JET, reduced particle confinement and increased SOL density scale length during monopole H-modes were attributed to rf-induced convection. ${ }^{20}$ In Tore Supra the up/down heat flux asymmetry on the antenna was interpreted as arising from a largescale rf-sheath driven convection roll pattern in front of the antenna. ${ }^{23-25}$ This convection occurs because the antenna acts like a giant biased probe, charging positive all the field lines in front of it. The tokamak magnetic field gives a preferred direction to the $\mathbf{E} \times \mathbf{B}$ drift pattern and is responsible for convecting plasma preferentially into the bottom of the antenna. Recently, ${ }^{23}$ it has been demonstrated that this heat flux asymmetry reverses with reversal of the tokamak B-field, consistent with the rf-driven convection mechanism (although power flow asymmetries due to the Hall term may also play a role ${ }^{26}$ ). Convective physics modifies fluxes into the antenna, affects sputtering, electron sheath heating (not discussed here) and, importantly, modifies the electron density profile in front of the antenna. Reflectometers were used to measure this effect on TFTR, 27 and show that the antenna effectively pumps on the edge plasma.

Thus, the rf antennas modify the edge density profile that they have to couple to. To treat this interaction theoretically, we consider the time-averaged vorticity or chargebalance equation,

$$
\frac{\mathrm{c}^{2}}{4 \pi \mathrm{v}_{\mathrm{a}}^{2}} \frac{\mathrm{d}}{\mathrm{dt}} \nabla_{\perp}^{2} \Phi=\nabla_{\|} \mathrm{J}_{\|}+\frac{2 \mathrm{c}}{\mathrm{B}} \mathbf{b} \times \kappa \cdot \nabla \mathrm{p}
$$


where $\mathrm{v}_{\mathrm{a}}$ is the Alfvén velocity and $\kappa$ is the curvature. The currents which contribute to the dynamics in Eq. (2) are ion polarization currents across the magnetic field, parallel currents which terminate on sheaths, and magnetic field line curvature. The perpendicular polarization current, which appears as the charge advection term on the left of Eq. (2), couples flux tubes in the perpendicular direction. The parallel current term describes the 1-D sheath dynamics considered earlier. The curvature term, usually neglected in rf physics, is what gives rise to low frequency edge turbulence. The edge instabilities driven by this term eject filaments of plasma called blobs into the scrape-off-layer (SOL). These blobs convect towards the antenna by a simple mechanism. 28,29 Curvature drift creates a charge separation. This gives rise to an internal electric field inside the plasma blob. The blob then convects radially as a whole due to the $\mathbf{E} \times \mathbf{B}$ drift. Consequently, the subject of antenna-plasma interaction is entwined with that of blobs and edge turbulence, and this interaction is fundamental to calculating the self-consistent SOL density profile in front of the antenna. ${ }^{16}$ This self-consistent density is required for studies of rf coupling, impurities, antenna damage and other antenna interaction effects.

Although the total power going into rf sheaths is most problematic at high power, at low power, this same effect can be used to diagnose rf sheaths. Since, the sheath power dissipation is linear with the voltage for $\mathrm{eV} / \mathrm{T}_{\mathrm{e}}>1$ [see Eq. (1)], its contribution to the loading resistance in this (low but not too low) power regime scales like $1 / \mathrm{V}$. Thus, sheath power can dominate the loading - a useful result for diagnosing the existence and properties (area, local density and voltage) of rf sheaths experimentally and potentially for validation of antenna-sheath codes. This effect has been observed $^{30}$ and successfully modeled ${ }^{10}$ to show that sheath area, voltage and ambient density are the most important parameters.

The ubiquity of antenna sheaths has motivated work into sheath mitigation by the use of insulating materials. ${ }^{31}$ On Phaedrus it was shown that the plasma potential rise due to rf sheath rectification could be almost completely eliminated by employing insulating limiters to intercept the field lines before they contact the metal, and complete the sheath circuit.32 Effectively, the insulator adds an additional series impedance to the plasma sheath and absorbs most of the voltage drop that would otherwise appear across the sheaths. The main challenge here will be to come up with insulating materials that can withstand a reactor environment. Boron compounds are often used in present day experiments, but novel ceramic materials have also been investigated. 33,34

When field lines are sufficiently long (so that the plasma resistance supports a significant voltage drop along the field line) the sheaths at the two ends become "disconnected". When these sheaths are also asymmetric (different voltages), they can drive a net dc parallel current. This effect was studied on TEXTOR, ${ }^{35}$ and more recently on $\mathrm{JET}^{36}$ where it was found that the sheath driven currents can trigger arcs at the high voltage end in some situations. This occurred in mixed phasing experiments where there was a current path between powered monopole and dipole antennas. In this case the cross-field polarization current driven by rf convection was postulated to be part of the current path. 
So far the discussion has been confined to sheath losses local to the antennas. But edge parasitic power losses are often observed in low single-pass and low- $\mathrm{k}_{\|}$phasing situations where near field sheaths do not appear to explain the whole story. One concept which can relate very well to this type of observation is that of the far field sheath, which gives a general mechanism for dissipation of wave energy in the SOL.

Edge rf fields appear on walls and limiters due to poor single pass absorption, or direct coupling to edge and surface modes. ${ }^{8}$ Because the flux surfaces are not generally aligned with conducting boundaries, the FW polarization alone cannot satisfy the proper boundary conditions, and of necessity a slow wave with $E_{\|}$is generated. ${ }^{37,38}$ This slow wave is often evanescent. The presence of this $E_{\|}$in the boundary plasma brings into play all of the sheath effects that have been discussed so far in the near field antenna context. In particular far field sheaths give a mechanism for edge power loss and impurity generation. Other dissipation mechanisms for waves at the edge are also possible, for example collisional dissipation of wave energy ${ }^{8}$ by neutral collisions. The low- $\mathrm{k}_{\|}$modes, being less evanescent in their propagation from the core towards the walls, are most susceptible to these dissipation mechanisms.

\section{IBW EDGE INTERACTIONS}

Sheaths can be just as important for IBW edge interactions as in the FW case of the preceding section. However, the IBW case also allows a rich variety of other nonlinear physics, primarily because Bernstein waves have a small group velocity and consequently require large electric fields to carry a significant power flux. The linear theory of IBW coupling is rather well developed and has been reviewed by $\mathrm{Ono}^{3}$ where interesting experimental results and nonlinear mechanisms are also reported. IBW coupling has met with mixed success and linear theory alone fails to describe many experiments. Coupling of power to the core has generally been better on small machines, and experiments benefit from good conditioning. In a number of cases, the application of IBW power has failed to heat the core plasma at all. Here we review some nonlinear effects which bear on the issue of getting IBW power through the SOL into the core, noting that in some cases the same physics can also be relevant to nominal FW and high harmonic fast wave (HHFW) experiments where the large pitch of $B$ relative to the antenna current strap results in substantial $E_{\|}$(slow wave) coupling.

Ponderomotive expulsion of plasma is one of the expected nonlinear mechanisms. For the slow wave, the (repulsive) ponderomotive potential is usually approximated by the jitter energy of electrons in the parallel rf field,

$$
\Psi \sim \frac{1}{2} \mathrm{~m}_{\mathrm{e}} \tilde{\mathrm{u}}_{\|}^{2}=\frac{\mathrm{e}^{2} \mathrm{E}_{\|}^{2}}{4 \mathrm{~m}_{\mathrm{e}} \omega^{2}}
$$

For representative electric fields $\mathrm{E}_{\|} \sim 300 \mathrm{~V} / \mathrm{cm}$ and frequencies in the range of tens to hundred of $\mathrm{MHz}$, the condition of strong nonlinear interactions, $\Psi>\mathrm{T}$ (e.g. in the SOL or at the separatrix) is easily met in all but the highest frequency experiments. ${ }^{3}$ Measurements from the DIII-D tokamak ${ }^{39}$ showed that as the power is raised the 
effect on the reactive loading is the same as moving the plasma away from the antenna, and is consistent with a ponderomotive expulsion interpretation.

Many large-tokamak IBW experiments have shown that the loading resistance is large and insensitive to the frequency (i.e. to the location of cyclotron resonance with respect to the antenna). This feature, was not expected from traditional direct launch IBW theory, but could be explained by a linear theory model 40 which assumed ponderomotive depletion of density in front of the antenna, and allowed the energy to be absorbed at the ensuing lower hybrid resonance (LHR). A related 1-D nonlinear model, ${ }^{41}$ which explicitly included ponderomotive profile steepening, showed enhanced wave reflection near the LHR that effectively channeled energy into a coaxial mode propagating in the halo plasma. Furthermore, the phasing properties of this mode were consistent with loading and heating efficiency measurements on TFTR. ${ }^{42-44}$ The basic idea is that the longer poloidal wavelength in 0-0 poloidal phasing enables a shorter radial wavelength parasitic coaxial mode to fit in the halo plasma between the LHR and the wall. These observations may underlie some of the different IBW behaviors in large and small machines.

In IBW experiments, parametric decay instability (PDI) is often observed. 45 Observations of PDI are often correlated with edge ion heating, as noted recently in the HHFW context on NSTX. 46 Parametric decay may be important in deciding what happens to wave energy that is trapped in the edge; however, it has been difficult to measure the power going into the PDI daughter waves.

In the parametric decay interaction, there is a large amplitude pump wave at frequency and wavenumber $\left(\omega_{0}, \mathbf{k}_{0}\right)$, and it is presumed that there are two other modes in the plasma at frequencies and wavenumbers which add up to $\left(\omega_{0}, \mathbf{k}_{0}\right)$, called the daughter waves, denoted by $(\omega, \mathbf{k}),\left(\omega_{-}, \mathbf{k}_{-}\right)$. The wave equation for each daughter mode is driven by a nonlinear beat current of the other daughter with the pump,

$$
\left[(\mathrm{c} / \omega)^{2} \nabla \times \nabla \times-\varepsilon \cdot\right] \mathbf{E}(\omega) \propto \mathbf{E}\left(\omega_{0}\right) \mathbf{E}\left(\omega_{-}\right)
$$

The initially small daughter waves can be linearly unstable above a certain threshold pump wave amplitude, which typically depends on the damping rates of the daughter modes. This instability is the PDI.

In the so-called dipole approximation (i.e. that of a long wavelength pump), the theoretical analysis can be done linearly by transforming to an oscillating frame, viz. the frame of the jitter in the pump wave field. In this case the species-dependent jitter is what provides the mode coupling that make the PDI process unstable. This linear theory of PDI (that is with a fixed pump wave) is rather well developed for the FW and IBW cases of interest for fusion plasmas. ${ }^{47}$ These types of calculations can also take into account convection of wave energy out of the interaction region in inhomogeneous plasmas. In FTU it was shown ${ }^{48}$ that there are competing constraints on the optimal SOL density: high to reduce PDI, but low to reduce reflected power for good linear coupling.

Theoretically, fully nonlinear calculations (including pump depletion) are very difficult. For IBW, the theory must include kinetic, hot plasma dynamics, and two or three spatial dimensions for realistic results. This problem presents an opportunity for future theory and simulation. 


\section{SLOW WAVE CORE INTERACTIONS: FLOW DRIVE}

In many experiments spanning several decades, it was found that directly launched IBW power could trigger improved confinement regimes in a tokamak. $49-55$ In other experiments, there were observations of IBW-induced flows.56,57 Collectively, experiments show that the IBW can drive flows, and that the IBW can sometimes enhance confinement, however the mechanisms have not been fully established experimentally.

Plasma turbulence research has shown that sheared flows can suppress turbulence.58-60 This knowledge has stimulated theoretical work on the calculation of rf driven flows, beginning with the pioneering work of Craddock and Diamond 61 and continuing up to the present. ${ }^{48,62-69}$ A number of 1-D and ray tracing calculations established that local absorption of IBW power at a cyclotron resonance was accompanied by redistribution of momentum that resulted in sheared poloidal (bipolar) flows.

Theoretically and experimentally, the direct launch IBW scheme for flow drive and turbulence suppression appears to be plausible, but practically it can be difficult to get the IBW power into the plasma, as noted in the previous section. This raises the question of whether mode-converted slow waves such as the IBW or ICW could be used to drive flows while avoiding the problems associated with direct IBW launch.

When the FW encounters a mode conversion (MC) layer in a multi-ion-species plasma, both the IBW and the ICW can result as mode conversion products. $68,70,71$ New diagnostics, such as phase contrast imaging have allowed these waves to be observed directly, ${ }^{72}$ and have helped to stimulate new theoretical work on flow drive, generalizing the previous work to handle MC, hot plasmas, and general electromagnetic waves.

There are basically three mechanisms by which an RF wave can induce forces on a plasma. The first one can be thought of as photon absorption, in which the rf wave energy is absorbed and imparts a proportional momentum, $\mathbf{k} / \omega$, to the plasma. This process is most effective for slow waves, with their relatively large $\mathbf{k}$. Note that this is fundamentally a dissipative force.

The second mechanism can be described as photon reflection. In the extreme case of total reflection, the force is $2 \mathbf{k} / \omega$ times the one-way power flow. However, this mechanism is better thought of in terms of reactive ponderomotive forces, driven by the gradient of the electric field amplitude rather than as related to circulating power. It is fundamentally non-dissipative.

The third mechanism is a momentum redistribution mechanism related to the Reynolds stress. No net force can be supplied by this mechanism, but adjacent flux surfaces can acquire equal and opposite forces and thereby create sheared flows. The nonlinear stress tensor which describes this process contains both the mechanical Reynolds stress component $\mathbf{v v}$ and the electromagnetic stress $\mathbf{B B}$. For an electromagnetic wave there can sometimes be cancellations between the two pieces. 61,66

There are several elegant formalisms for calculating nonlinear plasma effects due to rf waves, including guiding center ${ }^{73}$ and quiver kinetics ${ }^{74}$ formulations. Recently, 
flow drive work 64,69 has been developed using a different formulation, that of the $\mathrm{W}$ matrix ${ }^{75,76}$ developed to describe energy flow and absorption in the presence of nonlocality introduced by finite gyroradius effects. This formalism uses a global Fourier representation of the rf fields, and is well suited to implementation in Fourier based codes. ${ }^{77}$ The matrix $\mathrm{W}\left(\mathbf{k}, \mathbf{k}^{\prime}\right)$ is the generalization of the usual hot plasma conductivity matrix $\sigma(\mathbf{k})$ to the nonlocal case. Thus the familiar $\mathbf{J} \cdot \mathbf{E}$ expression for absorbed power is generalized to

$$
\mathrm{P}_{\mathrm{rf}}=\frac{1}{4} \sum_{\mathrm{kk}^{\prime}} \mathrm{e}^{\mathrm{i}\left(\mathbf{k}^{\prime}-\mathbf{k}\right) \cdot \mathbf{r}} \mathbf{E}_{\mathrm{k}}^{*} \cdot \mathrm{W}\left(\mathbf{k}, \mathbf{k}^{\prime}\right) \cdot \mathbf{E}_{\mathrm{k}^{\prime}}+\mathrm{cc}
$$

Analogous to the energy moment of the Vlasov equation from which $\mathrm{P}_{\mathrm{rf}}$ arises, one can take the momentum moment. In this case the nonlinear driving terms are the forces, which include the Lorentz force, and the divergence of a nonlinear stress tensor which involves the second order distribution function. It is shown in Ref. 69 that the total force on a fluid element can be expressed in terms of the three basic mechanisms, direct absorption, reactive ponderomotive force, and momentum redistribution. The reactive ponderomotive term reduces exactly to well known expressions in the fluid limit. Furthermore, flux-surface-averaged plasma flows in a tokamak can be driven only by the dissipative forces. Remarkably, these may be expressed simply in terms of the $\mathrm{W}$ matrix. The direct absorption term can drive net flows, depends on the momentum in the waves, and is effective with either electron or ion dissipation. The dissipative stress (momentum redistribution) term drives bipolar sheared flows but no net flows. It depends on the power absorbed in the perpendicular direction and scales inversely with the cyclotron frequency, so it is only significant for ions. In general flow drive is largest for short wavelengths and narrow dissipation layers, the narrow layers implying stronger shear in the flow.

This flow drive theory was implemented in the AORSA code 68 and applied to a CMod mode-conversion case which generates both IBW and ICW products. The toroidal flow can be obtained by balancing the rf force with an empirical diffusion of toroidal angular momentum. For $1 \mathrm{MW}$ of power, the flow is in the range of a few $\mathrm{km} / \mathrm{s}$ and the peak shearing rate is about $10^{4} \mathrm{~s}^{-1}$, which is somewhat small for effective turbulent suppression. To date, a careful survey of parameter space for more optimal cases has not been done. Also, there are some subtleties in the converting forces to flows, that bring in both neoclassical and turbulent transport theory. More theoretical work is needed in this area, particularly including time transients and anomalous diffusion which couple the poloidal and toroidal flows in the theory. Experiments that exhibit rf-induced confinement improvement and have the diagnostic capability to make measurements of poloidal and toroidal velocity shear are also needed, as well as experimental validation of flows from mode-converted waves.

Turbulence suppression is approximated governed by the shear in the radial electric field. There are different mechanisms for modifying $\mathrm{E}_{\mathrm{r}}$ shear by applied rf waves that can be seen from the steady state ion radial force balance equation.

$$
\frac{\mathrm{v}_{\zeta} \mathrm{B}_{\theta}-\mathrm{v}_{\theta} \mathrm{B}_{\zeta}}{\mathrm{RB}_{\theta}} \equiv \mathrm{G}(\psi)=-\mathrm{c}\left(\frac{\partial \Phi}{\partial \psi}+\frac{1}{\mathrm{Zen}_{\mathrm{i}}} \frac{\partial \mathrm{p}_{\mathrm{i}}}{\partial \psi}\right)+\left\langle\frac{\mathrm{c}}{\mathrm{Zen}_{\mathrm{i}}} \frac{\mathrm{F}_{\mathrm{i} \psi}}{\mathrm{RB}_{\theta}}\right\rangle_{\psi}
$$


The flux function $G(\psi)$, representing the $\mathbf{v} \times \mathbf{B}$ flow term, balances the radial electric field, the ion pressure gradient and any external radial forces. Nonlinear wave momentum processes drives flows, as discussed in the preceding paragraphs; the rf can modify the ion pressure profile locally, changing $\nabla p_{i}$; and finally, in principle, the waves can exert a direct radial ponderomotive force, although in practice this is almost always negligible in large tokamaks.

\section{INTEGRATED MODELING: THE NEW FOREFRONT}

The preceding issues have implications for integrated modeling, which is an exciting new forefront for rf physics. Integrated modeling can play an important role in hardware design, scenario development (including not only core rf physics, but now also nonlinear edge rf physics) and in the interpretation of experimental results.

One promising area is the incorporation of more edge physics into antenna coupling codes, such as plasma (blobs and turbulence) in the antenna region, wave scattering from blobs and fluctuations, sheath and ponderomotive effects and surface physics (e.g. sputtering and neutral gas desorption).

Inclusion of this physics would provide a predictive capability for plasma loading with a self-consistent density profile. At present, there is no robust way of predicting in advance the antenna loading for future experiments, mainly because the edge and SOL density profile is not known. Additionally, this type of integrated modeling could predict some operational constraints on the antenna such as local power dissipation, hot spot damage, and possibly certain types of arcs. An exciting goal would be a complete self-consistent description of the effects of rf on the edge (e.g. turbulence), and visa versa. Some work in this direction is in progress. ${ }^{14,16,23}$

For such a computational project to succeed, validation of codes with experiments at the most fundamental level is necessary. Low power loading measurements 30 would provide a very useful tool in this regard, as well as yielding a direct experimental diagnosis of sheaths, local plasma density, and antenna-plasma interactions.

A second promising area for integrated modeling is that of more realistic edge conditions for global full wave rf codes. Typically in these codes, all the launched power is absorbed in the core no matter how weak the core absorption is. It is known from experiments that edge physics is especially important for low $\mathrm{k}_{\|}$cases. More realistic models of edge dissipation are needed, for example employing boundary conditions to model sheaths. ${ }^{13-16}$ Edge collisions and neutrals may also be important in some cases. Incorporation of the missing edge physics will allow a new predictive capability for lost power and heating efficiency.

In conclusion, nonlinear effects are generally important for ICRF waves at the edge and nonlinear effects can also be important in the core for short wavelength, slow waves such as the IBW and ICW. Many important individual pieces of nonlinear RF interactions are at least partially understood as isolated phenomena. These include rfsheaths and their role on impurities, convection, SOL currents, ponderomotive effects, far field sheaths and edge dissipation, parametric decay, and rf effects on plasma flows and $\mathrm{E}_{\mathrm{r}}$. In order to make this knowledge really useful in a practical way, more 
integration of these pieces is needed. Integrated rf-edge modeling holds out the exciting possibility of a predictive capability that has so far been elusive.

As the fusion community stands at the threshold of a burning plasma experiment, our motivation for this new predictive capability is strong. Furthermore, we are acquiring the means for such computations through grand challenge computing resources that can make these computations feasible, both from a hardware and software perspective. These circumstances provide a significant opportunity for the ICRF theory and simulation community.

\section{ACKNOWLEDGMENTS}

This work was supported by U.S. DOE grant DE-FG02-97ER54392. Discussions with R. I. Pinsker, L. Colas and B. P. LeBlanc are acknowledged as well as the involvement and support of the RF SciDAC Team.

\section{REFERENCES}

1. J.-M. Noterdaeme, AIP Conf. Proc. 244, 71 (1992).

2. J.-M. Noterdaeme and G. Van Oost, Plasma Phys. Control. Fusion 35, 1481 (1993).

3. M. Ono, Phys. Fluids B 5, 241 (1993).

4. M. Bures, J. Jacquinot, K. Lawson, M. Stamp, et al., Plasma Phys. Control. Fusion 33, 937 (1991).

5. L. Colas, E. Faudot, S. Bremond, S. Heuraux. et al., this conference.

6. H. S. Butler and G. S. Kino, Phys. Fluids 6, 1346 (1963).

7. F. W. Perkins, Nucl. Fusion 29, 583 (1989).

8. M. Brambilla, R. Chodura, J. Hoffmann, J. Neuhauser, Plasma Phys. Control. Nucl. Fusion Res. 1990 (IAEA, Vienna, 1991), Vol. 1, p. 723.

9. J. R. Myra, D. A. D’Ippolito and M. J. Gerver, Nuclear Fusion 30, 845 (1990).

10. D.A. D’Ippolito and J. R. Myra, Phys. Plasmas 3, 420 (1996).

11. G.J. Greene, Ph. D. dissertation, California Institute of Technology, Pasedena, CA, 1984.

12. M.A. Lieberman, IEEE Trans. Plasma Sci. PS-16, 638 (1988).

13. E.F. Jaeger, L.A. Berry, J.S. Tolliver and D.B. Batchelor, Phys. Plasmas 2, 2597 (1995).

14. M. D. Carter, D. A. D'Ippolito, J. R. Myra and D. A. Russell, this conference.

15. J.R. Myra, D. A. D’Ippolito and M. Bures, Phys. Plasmas 1, 2890 (1994).

16. D. A. D’Ippolito, J. R. Myra, D. A. Russell and M. D. Carter, this conference.

17. S.J. Wukitch., R.L. Boivin, P.T. Bonoli, et al., Plasma Phys. Control. Fusion 46, 1479 (2004).

18. D.A. D’Ippolito, J.R. Myra, M. Bures, and J. Jacquinot, Plasma Phys. Cont. Fusion 33, 607 (1991).

19. J.R. Myra, D.A. D’Ippolito and Y.L. Ho, Fusion Eng. Design 31, 291 (1996).

20. D.A. D’Ippolito, J.R. Myra, J. Jacquinot and M. Bures, Phys. Fluids B 5, 3603 (1993).

21. R.A. Moyer, R. Van Niewenhove, G. Van Oost, et al., J. Nucl. Mater 176-177, 293 (1991).

22. D. Diebold, R. Majeski, T. Tanaka, et al., Nucl. Fusion 32, 2040 (1992).

23. L. Colas, L. Costanzo, C. Desgranges. et al., Nucl. Fusion 43, 1 (2003); L. Colas, E. Faudot, S. Brémond, S. Heuraux, this conference.

24. M. Bécouldet, L. Colas, S. Pécoul et al., Phys. Plasmas 9, 2619 (2002).

25. E. Faudot, S. Heuraux and L. Colas, this conference.

26. E.F. Jaeger, M.D. Carter, L.A. Berry, D.B. Batchelor, et al., Nucl. Fusion 38, 1 (1998).

27. D. A. D’Ippolito, J. R. Myra, J. H. Rogers, K. W. Hill, et al., Nucl. Fusion 38, 1543 (1998).

28. S. I. Krasheninnikov, Phys. Lett. A 283, 368 (2001).

29. D. A. D’Ippolito, J. R. Myra, and S. I. Krasheninnikov, Phys. Plasmas 9, 222 (2002).

30. D. W. Swain, R. I. Pinsker, F. W. Baity, M. D. Carter, et al., Nucl. Fusion 37, 1 (1997).

31. R. Majeski, P. H. Probert, T. Tanaka, D. Diebold, et al., Fusion Eng. Design 24, 159 (1994). 
32. J. Sorensen, D. A. Diebold, R. Majeski, N. Hershkowitz, Nucl. Fusion 36, 173 (1996).

33. J. R. Myra, D. A. D’Ippolito, J. A. Rice and C. S. Hazelton, J. Nucl. Mater 249, 190 (1997).

34. D. A. D’Ippolito, J. R. Myra, J.A. Rice and C.S. Hazelton, AIP Conf. Proc. 403, 463 (1997).

35. R. Van Nieuwenhove and G. Van Oost, Plasma Phys. Control. Fusion 34, 525 (1992).

36. D. A. D’Ippolito, J. R. Myra, P. M. Ryan, E. Righi, et al., Nucl. Fusion 42, 1357 (2002).

37. F.W. Perkins, Bull. Am. Phys. Soc. 34, 2093 (1989), paper 6 S6.

38. J.R. Myra and D. A. D’Ippolito, Phys. Plasmas 1, 2890 (1994).

39. M. J. Mayberry, R. I. Pinsker, C. C. Petty, M. Porkolab, et al., Nucl. Fusion 33, 627 (1993).

40. S.C. Chiu, M.J. Mayberry, R.I. Pinsker, C.C. Petty, M. Porkolab, AIP Conf. Proc. 244, 169 (1992).

41. D.A. Russell, J.R. Myra and D.A. D’Ippolito, Phys. Plasmas 5, 743 (1998).

42. J.R. Wilson, R.E. Bell, S. Bernabei, et al., Phys. Plasmas 5, 1721 (1998).

43. J.R. Myra, D. A. D’Ippolito, D.A. Russell, J.H. Rogers, T. Intrator, Phys. Plasmas 7, 283 (2000).

44. T. Intrator, J.R. Myra and D. A. D’Ippolito, Nucl. Fusion 43, 531 (2003).

45. R.I. Pinsker, C.C. Petty, M.J. Mayberry, M. Porkolab, et al., Nucl. Fusion 33, 777 (1993).

46. J.R. Wilson, this conference.

47. M. Porkolab, Fusion Eng. Design 12, 93 (1990).

48. A. Cardinali, C. Castaldo, R. Cesario, et al., Nucl. Fusion 42, 427 (2002).

49. M. Ono, P. Beiersdorfer, R. Bell, S. Bernabei, et al., Phys. Rev. Lett. 60, 294 (1988).

50. T. Seki, K. Kawahata, M. Ono, K. Ida, et al., AIP Conf. Proc. 244, 138 (1991).

51. J. D. Moody, M. Porkolab, C. L. Fiore, F. S. McDermott,et al., Phys. Rev. Lett. 60, 298 (1988).

52. B. LeBlanc, S. Batha, R. Bell, S. Bernabei, et al. Phys. Plasmas 2, 741 (1995).

53. C.K. Phillips, M.G. Bell, R.E. Bell, S. Bernabei, et al., Nucl. Fusion 40, 461 (2000).

54. R. Cesario, A. Cardinali, C. Castaldo, M. Leigheb, et al., Phys. Plasmas 8, 4721 (2001).

55. B Wan, Y. Zhao, J. Li, et al., Phys. Plasmas 10, 3703 (2003).

56. B.P. LeBlanc, R.E. Bell, S. Bernabei, J.C. Hosea, et al., Phys. Rev. Lett. 82, 331 (1999).

57. C. Riccardi, F. De Colle, M. Fontanesi, C. C. Petty, et al., AIP Conf. Proc. 595, 83 (2001).

58. H. Biglari, P.H. Diamond and P.W. Terry, Phys. Fluids B 2, 1 (1990).

59. K.H. Burrell, T.N. Carlstrom, E.J. Doyle, et al., Plasma Phys. Control. Fusion 34, 1859 (1992)

60. P.W. Terry, Rev. Mod. Phys. 72, 109 (2000).

61. G.G. Craddock and P.H. Diamond, Phys. Rev. Lett. 67, 1535 (1991).

62. M. Ono et al., Plasma Phys. Control. Nucl. Fusion Res. 1994 (IAEA, Vienna, 1995), Vol. 1, p. 469.

63. L.A. Berry, E.F. Jaeger and D.B. Batchelor, Phys. Rev. Lett. 82, 1871 (1999).

64. E. F. Jaeger, L. A. Berry, and D. B. Batchelor Phys. Plasmas 7, 3319 (2000).

65. A. G. Elfimov, G. Amarante-Segundo, et al. Phys. Rev. Lett. 84, 1200 (2000).

66. J.R. Myra and D.A. D’Ippolito, Phys. Plasmas 7, 3600 (2000).

67. H. Weitzner, L.A. Berry, E. F. Jaeger and D. B. Batchelor, Phys. Plasmas 7, 564 (2000).

68. E.F. Jaeger, L.A. Berry, J.R. Myra, D.B. Batchelor, et al., Phys. Rev. Lett. 90, 195001 (2003).

69. J. R. Myra, L. A. Berry, D. A. D’Ippolito, and E. F. Jaeger, Phys. Plasmas 11, 1786 (2004).

70. F.W. Perkins, Nucl. Fusion 17, 1197 (1977).

71. E. Nelson-Melby et al., Phys. Rev. Lett. 90, 155004 (2003).

72. Y. Lin, this conference; and refs. therein.

73. J.R. Cary and A.N. Kaufman, Phys. Fluids 24, 1238 (1981).

74. P. J. Catto et al., Phys. Fluids B 2, 2395 (1990).

75. D.N. Smithe, Plasma Phys. Control. Fusion 31, 1105 (1989).

76. M. Brambilla, Kinetic Theory of Plasma Waves, (Clarendon Press, Oxford, 1998).

77. E.F. Jaeger, L.A. Berry, E.D. D’Azevedo, et al., Phys. Plasmas 8, 1573 (2001). 